\title{
The theory of planned behaviour and discrete food choices: a systematic review and meta-analysis
}

\author{
Máirtín S. McDermott ${ }^{1,6^{*}}$, Madalyn Oliver ${ }^{1}$, Alexander Svenson ${ }^{1}$, Thomas Simnadis², Eleanor J. Beck², Tim Coltman ${ }^{3}$, \\ Don Iverson ${ }^{4}$, Peter Caputi ${ }^{5}$ and Rajeev Sharma ${ }^{1}$
}

\begin{abstract}
The combination of economic and social costs associated with non-communicable diseases provide a compelling argument for developing strategies that can influence modifiable risk factors, such as discrete food choices. Models of behaviour, such as the Theory of Planned Behaviour (TPB) provide conceptual order that allows program designers and policy makers to identify the substantive elements that drive behaviour and design effective interventions. The primary aim of the current review was to examine the association between TPB variables and discrete food choice behaviours. A systematic literature search was conducted to identify relevant studies. Calculation of the pooled mean effect size $\left(r_{+}\right)$was conducted using inverse-variance weighted, random effects meta-analysis. Heterogeneity across studies was assessed using the Q- and $\mathrm{I}^{2}$-statistics. Meta-regression was used to test the impact of moderator variables: type of food choice behaviour; participants' age and gender. A total of 42 journal articles and four unpublished dissertations met the inclusion criteria. TPB variables were found to have medium to large associations with both intention and behaviour. Attitudes had the strongest association with intention $\left(r_{+}=0.54\right)$ followed by perceived behavioural control $\left(\mathrm{PBC}, r_{+}=0.42\right)$ and subjective norm (SN, $\left.r_{+}=0.37\right)$. The association between intention and behaviour was $r_{+}=0.45$ and between PBC and behaviour was $r_{+}=0.27$. Moderator analyses revealed the complex nature of dietary behaviour and the factors that underpin individual food choices. Significantly higher PBC-behaviour associations were found for choosing health compromising compared to health promoting foods. Significantly higher intention-behaviour and PBC-behaviour associations were found for choosing health promoting foods compared to avoiding health compromising foods. Participant characteristics were also found to moderate associations within the model. Higher intention-behaviour associations were found for older, compared to younger age groups. The variability in the association of the TPB with different food choice behaviours uncovered by the moderator analyses strongly suggest that researchers should carefully consider the nature of the behaviour being exhibited prior to selecting a theory.
\end{abstract}

Keywords: Discrete food choice, Theory of reasoned action, Theory of planned behaviour, Systematic review, Meta-analysis

\section{Background}

Non-communicable diseases such as cardiovascular disease, cancers and diabetes accounted for over two thirds of global deaths in 2012 [1] and are predicted to account for up to US $\$ 46.7$ trillion in cumulative losses

\footnotetext{
* Correspondence: mairtin@uow.edu.au

${ }^{1}$ School of Computing and Information Technology, Faculty of Engineering and Information Sciences, University of Wollongong, Wollongong, NSW 2522, Australia

${ }^{6}$ Centre for Health and Social Research (CHaSR), Australian Catholic University, Melbourne, Victoria, Australia

Full list of author information is available at the end of the article
}

in productivity between 2011 and 2030 [2]. These combined economic and social costs provide a compelling argument for developing strategies to address the key modifiable risk factors of these conditions, such as dietary consumption [3]. Domestic health authorities [4-6] are aware of the importance of encouraging healthy eating patterns and have developed guidelines that seek to optimise the health and wellbeing of their citizens. These guidelines have a focus on eating 'core' food groups, such as vegetables, fruits and whole-grains while avoiding the consumption of foods that are classified as 'non- 
core' or 'discretionary', such as cakes, pastries and soft drinks.

The healthy eating guidelines are reflective of a broader shift within the discipline of nutrition, away from the effects of nutrients to a more holistic notion of the effects of specific foods in the context of a whole diet [7]. This transition aligns with the premise that discrete 'food' choices, i.e. those decisions made by individuals at the point of consumption, rather than 'nutrient' choices drive eating patterns. These decisions are of particular interest due to the persistence of poor food choices within the general population (e.g. [8]). The challenge is to encourage health promoting behaviours, such as choosing whole over refined grain cereal products, increasing fruit and vegetables and consciously limiting discretionary food intake in order to reduce the risk of chronic disease.

Understanding the underpinnings of these behaviours will provide invaluable assistance in the development of behaviour change interventions, which are more likely to be effective when based on sound theory [9]. The Theory of Planned Behaviour (TPB) [10], which is an extension of the earlier Theory of Reasoned Action (TRA) [11] is one of the models most commonly used to understand health behaviours such as these. The TPB asserts that the most proximal determinant of behaviour is the intention to perform that behaviour. Intentions, which are considered to indicate the amount of effort an individual is likely to devote to performing a behavior, are in turn determined by attitudes, an overall evaluation of the behaviour, subjective norms (SN), an evaluation of whether an individual feels significant others think he/ she should engage in the behaviour and perceived behavioural control (PBC), which represents an individual's perceptions of control over that behaviour. To the extent that it reflects actual control, $\mathrm{PBC}$ is also held to exert a direct effect on behaviour. Thus, according to the theory, individuals will have a strong intention to, for example, eat the recommended daily amount of vegetables, when they hold positive attitudes towards that behaviour, perceive social pressures from those whose opinions they value and feel capable of eating the recommended amount without difficulty. This intention, along with their perceptions of capability, determines the likelihood that they will perform this behaviour. The effect of all other influences, for example biological, environmental and cultural, are hypothesised to be mediated by the TPB [12].

The potential of the TPB as a model for understanding health behaviours was confirmed by a review conducted by McEachan et al. [13] which found that the theory accounted for between 14 and $24 \%$ of the betweenstudy variance in behaviour. However, this review combined studies examining discrete food choice behaviours such as eating fruit and vegetables or avoiding sugarsweetened drinks, with broader dietary patterns such as 'eating a healthy diet'. Thus, their analyses may have masked important differences. For example, previous research has found that the association between TPB variables and behaviour may differ between broad categories of behaviour such as 'healthy eating' and more specific behaviours, such as food choices [14]. These data are also lacking when considered from a clinical perspective. Although dietary guidelines provide an overview of broad dietary patterns that will achieve optimum health for populations, clinicians are faced with the difficult task of managing the myriad of discrete food choices that shape these eating patterns. A more nuanced examination of the literature is therefore warranted. The primary aim of the current study is to examine the association between TPB variables and discrete food choice behaviours.

This review also has a number of secondary aims. Primarily, these aim to elucidate the association between the TPB and food choice behaviours by examining whether associations are moderated by the type of food choice behaviour examined. Critics argue that TPB research is frequently applied to a broad range of behaviour without consideration of whether such application is valid given the nature of the behaviour in question. The TPB is generally considered to present a rational view of behaviour, determined largely through a process of deliberative appraisal. When examined from alternative theoretical perspectives, the extent to which these processes apply to some food choices is open to question.

For example, from the perspective of Temporal Self-Regulation Theory (TST) [15], the TPB is deficient for not considering the differential temporal weighting of anticipated contingencies of health behaviours. Health-compromising behaviours, such as choosing highly palatable, calorie dense or high-fat foods, are frequently associated with many benefits and few costs at the point of action, whereas the same behaviours are associated with long term costs and few if any long term benefits. In contrast, health-promoting behaviours, such as choosing lower energy density, nutrient rich foods, frequently work in the opposite manner. In line with TST, the strength of association between the deliberative processes captured by the TPB and behaviour is hypothesised to vary based on the temporal frame of the specific food choice $[16,15]$.

Our second set of analyses will examine the literature from the perspective of dual-process models of behaviour (e.g. [17, 18]). These models propose that behaviour is determined by two interacting, parallel systems whereby automatic, impulsive processes, are in competition with more deliberative, rational determinants of behaviour such as those described in the TPB. Given that health promoting food choices can be broadly 
categorised into one of two groups: (a) choosing health promoting foods that typically have a lower hedonic value than alternative foodstuffs, and may therefore be governed by deliberative processes; and (b) resisting the impulse to choose health compromising foods with high hedonic value, one might expect the association between the TPB and behaviour to be stronger for (a) than (b). In order to inform the development of interventions facilitating health-promoting food choices, we shall also examine this distinction here.

Finally, in order to generate further evidence that may facilitate the development of targeted interventions, we will also examine the impact of participant characteristics within the context of health promoting food choice behaviours. Specifically we will examine whether the associations specified within the TPB are moderated by age and gender. National survey data from Australia [8] has found important differences in food choices based on these characteristics. For example, that men are more likely to consume unhealthy foods such as soft drinks or burgers, and that that teenagers and younger adults are less likely to consume fruit compared to the general population, a pattern that is repeated worldwide [19].

\section{Review}

\section{Methods}

The design, conduct and reporting of this systematic review was informed by the Preferred Reporting Items for Systematic Reviews and Meta-Analyses guidelines (PRISMA [20]) (the PRISMA checklist is available as Additional file 1). As the study involved the secondary analysis of existing datasets, ethical approval was not sought. The funding organization for this study had no role in the collection, analysis and interpretation of data, or the right to approve the finished manuscript prior to publication. As the current study was conducted as part of a larger program of research, no study protocol was produced.

\section{Selection criteria}

In accordance with PRISMA, the PICOS (population, intervention, comparison, outcome, study design) approach [21] was used to formulate the selection criteria. Studies that explicitly applied the TRA or TPB to the choice of specific foods (e.g. high fibre bread, fruit, fish) or narrow categories of foods (e.g. high calorie snacks, dairy products, ready meals) were included. Studies investigating associations between these models and dietary patterns (e.g. healthy eating or eating a low-fat diet) were not included in the current review. These have been examined elsewhere [22]. Studies were included where participants were drawn from a population without any current or former psychiatric or medical condition, for example eating disorders or diabetes, as the psychological determinants of food choice behaviours in these populations may not be generalizable to the community at large. In line with previous reviews (e.g. [23]), studies were excluded if participants received an intervention. Studies were not selected based on any comparison between conditions. In line with theoretical models, TRA studies must at minimum have reported correlations between the following outcomes: attitudes and subjective norm with intentions, and intentions with behaviour. TPB studies must at minimum have reported correlations between attitudes, subjective norm and perceived behavioural control with intentions, and perceived behavioural control and intentions with behaviour. We included any quantitative study design provided the other inclusion criteria were met. In addition, studies needed to report sample size, full details (i.e. item wording, response scale and response anchor) of at least one of the items used to measure each variable and be published in the English language.

\section{Study identification}

We searched for published and unpublished research in PsycINFO, MEDLINE, Web of Science, CINAHL and ProQuest Dissertations \& Theses. Full details of the electronic search strategies used can be found in Additional file 2 . We also manually searched the reference lists of all studies selected for inclusion, and the reference list of a key systematic review [13]. Final searches were conducted in October 2014.

Two authors (MO \& MSMcD) pre-screened one half of the database containing all titles and abstracts for relevance. These studies were then selected for inclusion independently by the same two authors. Agreement was substantial $(\mathrm{K}=.80)$ [24]. Data from each study was extracted by one of two authors (MO, AS) who also independently coded each effect size for behaviour type. Again, agreement was substantial $(\kappa=.72)$.

\section{Data extraction}

In addition to correlations and sample size, we extracted data on gender, the mean age of participants, and the behaviour examined in each study. In order to test for the impact of temporal frame, each behaviour was coded as health promoting or health compromising. The health promoting category included studies where the food choice was deemed to have few benefits and potential costs (e.g. bland or unpleasant taste) at the point of action, but with benefits and few costs in the long term (e.g. reduction in risk for contracting chronic conditions such as coronary heart disease). Examples of behaviours coded into this category include choosing fruits and vegetables, high fibre bread and dairy products. The health compromising category contained studies examining behaviours that operated in the opposite manner, for 
example, choosing soft drinks, sugared snacks or junk food. Studies in which the temporal frame of the target behaviour was not clear were not included in these analyses.

Subsequently, we coded each health promoting behaviour into two further categories depending on whether the behaviour targeted choosing health promoting, or avoiding health compromising foods. Each decision was made based primarily on an examination of the items used to evaluate TRA/ TPB variables. For example, the study conducted by De Bruijn \& Van den Putte [25] assessed the consumption of soft drinks as the behavioural outcome (e.g. how many days per week do you consume sugar-sweetened soft drinks?), but the behaviour targeted by the cognitive items was to limit consumption of soft drinks (e.g. I intend to limit my amount of soft drink consumption'). The 'target behaviour' was therefore coded as avoiding the consumption of health compromising foods. A descriptive summary of each of the studies included in the review, including target behaviour and the coding each behaviour received can be found in Table 1 . A glossary of key terms and definitions used in the review is provided in Additional file 3.

A number of studies included in each meta-analysis provided multiple effect sizes that were eligible for inclusion. The decision of how to handle these data was guided by Borenstein et al. [26] and Sharma et al. [27]. In instances where multiple effect sizes were due to data being presented for independent samples or where data was presented from two or more time points using the same participants, these were treated as individual data points for analysis. Where multiple measurements of behaviour eligible for inclusion in the meta-analysis were reported in the same study, each was retained provided it yielded distinct information. Where data for 'higher order' TPB constructs (e.g. instrumental and descriptive norms) or individual items for constructs were reported separately, these were clustered to yield single correlations.

\section{Data analysis}

Calculation of the pooled mean effect size $\left(r_{+}\right)$was conducted using inverse-variance weighted random effects meta-analysis [26]. We also estimated the heterogeneity across studies, using both the $Q$ and $I^{2}$ statistics. To test for moderation we employed the protocol for random effects meta-regression recommended by Borenstein et al. [26]. All analyses were performed using Comprehensive Meta-Analysis (CMA) Version 3.0 [28].

\section{Results}

Search results

The electronic search strategy retrieved 10,238 unique records. A further five were identified through screening the reference lists of a related meta-analysis [13] and 31 through screening the reference lists of included articles.
In total, 42 journal articles and four dissertations met the inclusion criteria. Data from three studies were reported in more than one article ([29], [30, 31] and $[32,33])$. Relevant data were extracted from either article as appropriate. A total of 43 studies were therefore included. Full details of the screening process can be seen in the PRISMA Flow-Chart (Fig. 1).

\section{Association of TPB variables with intention and food choice}

Table 1 summarises the meta-analysed associations for TPB variables across studies. TPB variables were found to have medium to large associations with both intention and behaviour. Attitudes had the strongest association with intention $\left(r_{+}=0.54\right)$ followed by $\mathrm{PBC}\left(r_{+}=0.42\right)$ and $\mathrm{SN}\left(r_{+}=0.37\right)$. The association between intention and behaviour was $r_{+}=0.45$ and between PBC and behaviour was $r_{+}=0.27$. Forest plots for each association can be found in Additional file 4. Both the Q- and $I^{2}$-statistics revealed significant heterogeneity for each effect size (see Table 2) supporting the use of meta-regression to search for moderators.

\section{Test for moderation by behaviour type}

Mean effect sizes and heterogeneity statistics for each category of behaviour are shown in Table 3. As we were interested in the association between these behaviour types and assessments of behaviour, analyses were limited to intention-behaviour, and $\mathrm{PBC}$-behaviour associations. The first analysis compared health promoting with health compromising food choices from the perspective of TST. There was no evidence of moderation for the intention-behaviour association $\left(r_{+}=0.38\right.$ and $0.45, \chi^{2}(1)=2.59$, n.s.). However, there was a significant difference between health promoting and health compromising food choices for the $\mathrm{PBC}$-behaviour association $\left(r_{+}=0.31\right.$ and $\left.0.17, \chi^{2}(1)=8.23, p<0.01\right)$.

Following this, we tested whether there was evidence of moderation within health promoting food choices. On this occasion there was evidence of moderation for both the PBC-behaviour $\left(r_{+}=0.35\right.$ and $\left.0.21, X^{2}(1)=4.38, \quad p<0.05\right)$ and intention-behaviour associations $\left(r_{+}=0.43\right.$ and $\left.0.28, \chi^{2}(1)=7.93, p<0.01\right)$ for choosing health promoting and avoiding of health compromising food, respectively.

\section{Test for moderation by participant characteristics}

Table 4 shows the breakdown of effect sizes by mean age of participants. Age moderated the intention-behaviour association $\left(\chi^{2}(2)=9.22, p=0.01\right)$, with the $\leq 17$ age group differing significantly from the $30+$ age group $(B=-0.21$ [95\% CI: $-0.35,-0.06] p<0.01)$. There was no evidence that age moderated any of the other associations in the 
Table 1 Descriptive summary of studies included in the systematic review $(n=43)$

\begin{tabular}{|c|c|c|c|c|c|c|c|c|}
\hline Study & Article Type & Country & $\mathrm{N}$ & Gender & $\begin{array}{l}\text { Age } \\
\text { Category }\end{array}$ & Theory & Food choice & Behaviour coding \\
\hline Aghamolaei et al. (2012) [43] & Journal article & Iran & 321 & $62.9 \%$ & $\geq 30$ & TPB & Fish & NA \\
\hline $\begin{array}{l}\text { Astrom (2004) [44]/Astrom \& } \\
\text { Okullo (2004) [29] }\end{array}$ & Journal article & Uganda & 1146 & Not specified & $\leq 17$ & TRA & Sugared snacks & Choosing health compromising food \\
\hline Balian (2008) [45] & Dissertation & USA & 93 & $54.0 \%$ & $\leq 17$ & TPB & Milk, soda & $\begin{array}{l}\text { Choosing health compromising food } \\
\text { (Soda only) }\end{array}$ \\
\hline $\begin{array}{l}\text { Berg et al. (2000) [30]/Conner } \\
\text { et al. (2011) [31] }\end{array}$ & Journal article & Sweden & 1096 & $52.0 \%$ & $\leq 17$ & TPB & Milk, high fibre bread & $\begin{array}{l}\text { Choosing health promoting food } \\
\text { (High fibre bread only) }\end{array}$ \\
\hline Blanchard et al. (2009a) [46] & Journal article & USA & 511 & $49.7 \%$ & $18-29$ & TPB & Fruit \& vegetables & Choosing health promoting food \\
\hline Blanchard et al. (2009b) [47] & Journal article & USA & $176-237$ & $56.9 \%$ & $18-29$ & TPB & Fruit \& vegetables & Choosing health promoting food \\
\hline Branscum \& Sharma (2014) [48] & Journal article & USA & $69-98$ & $100.0 \% / 0.0 \%$ & $\leq 17$ & TPB & Snack foods, fruit \& vegetables & $\begin{array}{l}\text { Avoiding health compromising food } \\
\text { (snacks), choosing health promoting } \\
\text { food (fruit \& vegetables) }\end{array}$ \\
\hline Brug et al. (2006) [49] & Journal article & The Netherlands & 627 & $50.9 \%$ & $\geq 30$ & TPB & Fruit & Choosing health promoting food \\
\hline Churchill et al. (2008) [50] & Journal article & UK & 315 & $65.7 \%$ & $\geq 30$ & TPB & High-calorie snacks & Avoiding health compromising food \\
\hline Churchill \& Jessop (2011) [51] & Journal article & UK & 139 & $77.7 \%$ & $18-29$ & TPB & High-calorie snacks & Choosing health compromising food \\
\hline Collins \& Mullan (2011) [16] & Journal article & Australia & 190 & $77.9 \%$ & $18-29$ & TPB & Snacks, fruit \& vegetables & $\begin{array}{l}\text { Choosing health compromising food } \\
\text { (snacks), choosing health promoting } \\
\text { food (fruit \& vegetables) }\end{array}$ \\
\hline Corry (2008) [52] & Dissertation & USA & 159 & $53.5 \%$ & $18-29$ & TPB & Fruit \& vegetables & Choosing health promoting food \\
\hline De Bruijn (2010) [53] & Journal article & The Netherlands & 538 & $53.7 \%$ & $\geq 30$ & TPB & Fruit & Choosing health promoting food \\
\hline De Bruijn et al. (2012) [54] & Journal article & The Netherlands & 159 & $78.0 \%$ & $18-29$ & TPB & Fruit & Choosing health promoting food \\
\hline $\begin{array}{l}\text { De Bruijn \& Van den Putte } \\
\text { (2009) [25] }\end{array}$ & Journal article & The Netherlands & 312 & $65.3 \%$ & $\leq 17$ & TPB & Soft drinks & Avoiding health compromising food \\
\hline De Bruijn et al. (2009) [55] & Journal article & The Netherlands & 405 & $57.1 \%$ & $\geq 30$ & TPB & Fruit & Choosing health promoting food \\
\hline De Bruijn et al. (2007a) [56] & Journal article & The Netherlands & 521 & $53.7 \%$ & $\geq 30$ & TPB & Fruit & Choosing health promoting food \\
\hline De Bruijn et al. (2007b) [57] & Journal article & The Netherlands & 208 & $62.0 \%$ & $\leq 17$ & TPB & Soft drinks & Avoiding health compromising food \\
\hline De Bruijn et al. (2005) [58] & Journal article & The Netherlands & 3859 & $55.2 \%$ & $\leq 17$ & TPB & Snacks & Avoiding health compromising food \\
\hline $\begin{array}{l}\text { Karimi-Shahanjarini et al. } \\
\text { (2012) [59] }\end{array}$ & Journal article & Iran & 790 & $100.0 \%$ & $\leq 17$ & TPB & Junk food & Choosing health promoting food \\
\hline $\begin{array}{l}\text { Kassem (2000) [32]/Kassem } \\
\text { et al. (2003) [33] }\end{array}$ & Dissertation/Journal article & USA & 710 & $100.0 \%$ & $\leq 17$ & TPB & Milk, soft drinks & $\begin{array}{l}\text { Choosing health compromising food } \\
\text { (for soft drinks only) }\end{array}$ \\
\hline Kassem \& Lee (2004) [60] & Journal article & USA & 564 & $0.0 \%$ & $\leq 17$ & TPB & Soft drinks & Choosing health compromising food \\
\hline Kassem \& Lee (2005) [61] & Journal article & USA & 560 & $0.0 \%$ & $\leq 17$ & TPB & Milk & NA \\
\hline Kida \& Astrom (1998) [62] & Journal article & Tanzania & 309 & $46.5 \%$ & $\leq 17$ & TPB & Sugared snacks & Avoiding health compromising food \\
\hline Kim et al. (2003) [63] & Journal article & USA & 162 & $76.0 \%$ & $\geq 30$ & TPB & Dairy products & Choosing health promoting food \\
\hline
\end{tabular}


Table 1 Descriptive summary of studies included in the systematic review $(n=43)$ (Continued)

\begin{tabular}{|c|c|c|c|c|c|c|c|c|}
\hline Mahon et al. (2006) [64] & Journal article & UK & 1004 & $86.0 \%$ & Not specified & TPB & Ready meals, takeaways & $\begin{array}{l}\text { Choosing health compromising food } \\
\text { (ready meals \& takeaways) }\end{array}$ \\
\hline Masalu \& Astrom (2001) [65] & Journal article & Tanzania & 1090 & $32.0 \%$ & $18-29$ & TPB & Sugared snacks & Avoiding health compromising \\
\hline Mitterer-Daltoe et al. (2013) [66] & Journal article & Brazil & 200 & $60.0 \%$ & $\geq 30$ & TPB & Fish & NA \\
\hline Murnaghan et al. (2010) [67] & Journal article & Canada & 287 & $51.0 \%$ & $\leq 17$ & TPB & Fruit \& vegetables & Choosing health promoting food \\
\hline O'Neal et al. (2014) [68] & Journal article & USA & 211 & $73.0 \%$ & $\geq 30$ & TPB & Fruit \& vegetables & Choosing health promoting food \\
\hline Onwezen et al. (2014) [69] & Journal article & The Netherlands & 491 & $50.0 \%$ & $\geq 30$ & TPB & Fruit & Choosing health promoting food \\
\hline Povey et al. (2000) [14] & Journal article & UK & 151 & $70.0 \%$ & $\geq 30$ & TPB & Fruit \& vegetables & Choosing health promoting food \\
\hline Prell et al. (2002) [70] & Journal article & Sweden & 162 & $53.3 \%$ & $\leq 17$ & TPB & Fish & NA \\
\hline Richetin et al. (2008) [71] & Journal article & UK & 75 & $69.4 \%$ & $18-29$ & TPB & Soft drinks & Choosing health compromising food \\
\hline Sharifirad et al. (2013) [72] & Journal article & Iran & 521 & $46.8 \%$ & $\leq 17$ & TPB & Fast food & Choosing health compromising food \\
\hline Sjoberg et al. (2012) [73] & Journal article & USA & 258 & $80.6 \%$ & $\geq 30$ & TPB & Wholegrain bread & Choosing health promoting food \\
\hline Tak et al. (2011) [74] & Journal article & The Netherlands & 970 & $46.0 \%$ & $\leq 17$ & TPB & Sugar-sweetened beverages & Choosing health compromising food \\
\hline Tak et al. (2013) [75] & Journal article & The Netherlands & 323 & $54.1 \%$ & $\geq 30$ & TPB & Fruit & Choosing health promoting food \\
\hline Towler \& Shepherd (1991) [76] & Journal article & UK & 288 & $61.5 \%$ & $\geq 30$ & TRA & Chips & Choosing health compromising food \\
\hline Tuu et al. (2008) [77] & Journal article & Vietnam & 612 & $59.3 \%$ & $\geq 30$ & TPB & Fish & NA \\
\hline Verbeke \& Vackier (2005) [78] & Journal article & Belgium & 429 & $66.9 \%$ & $\geq 30$ & TPB & Fish & NA \\
\hline Verplanken (2006) [79] & Journal article & Norway & 128 & $64.1 \%$ & Not specified & TPB & Snacks & Choosing health compromising food \\
\hline Zoellner et al. (2012) [80] & Journal article & USA & 119 & $66.0 \%$ & $\geq 30$ & TPB & Sugar-sweetened beverages & Avoiding health compromising food \\
\hline
\end{tabular}

Notes: Gender was coded as the proportion of the sample that was female; Behaviour coding is provided for the behaviour targeted in each study. These were coded either as one of two types of health promoting food choice behaviour (choosing vs avoiding), or as a health compromising food choice behaviour. Behaviours coded as not applicable (NA) were not included in these analyses 


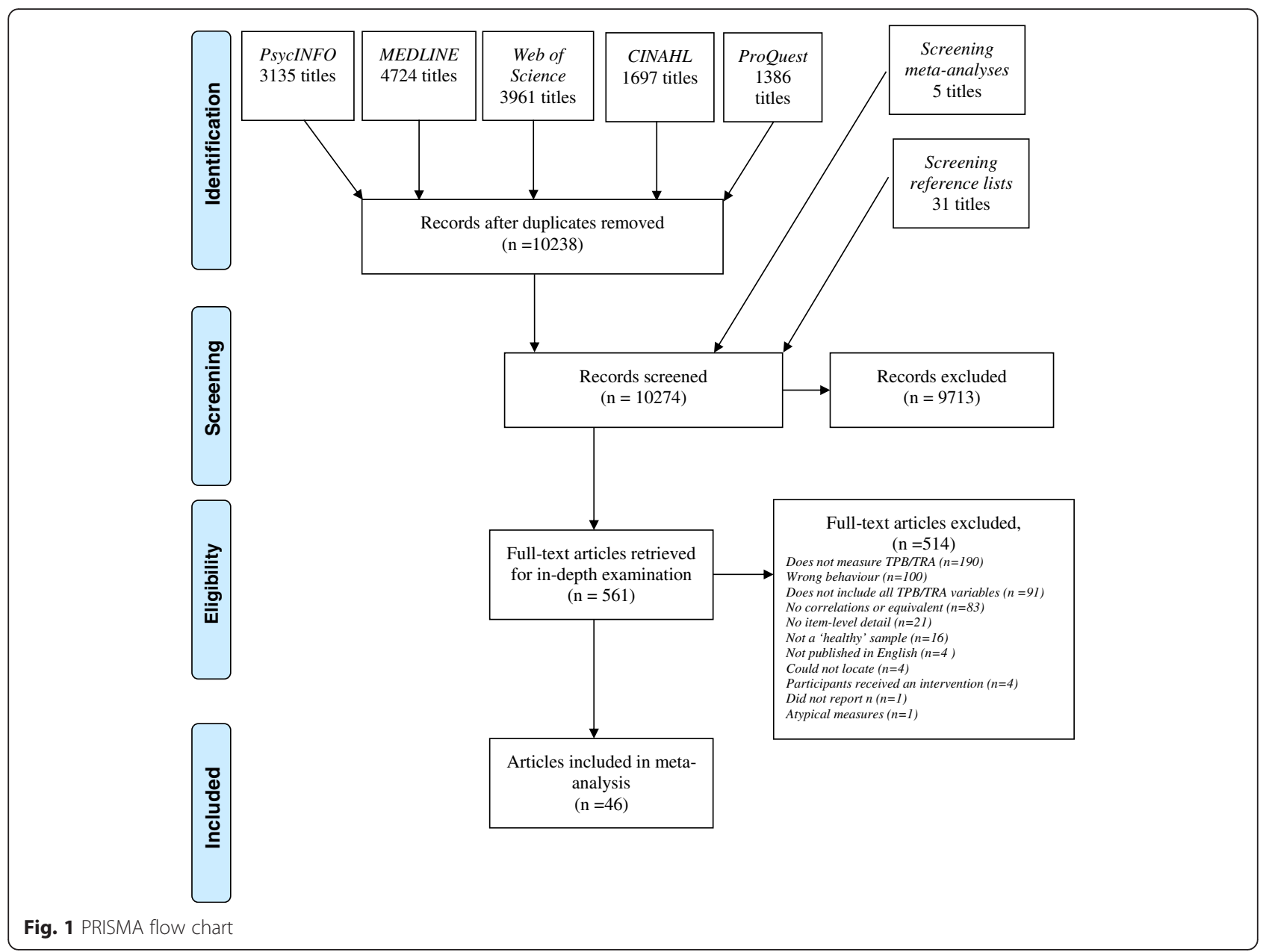

model. Gender was found not to moderate any of the associations within the TPB.

\section{Discussion}

To our knowledge, the current review is the first to examine the associations between TPB variables and food choice behaviours. In general, these associations were medium (SN and $\mathrm{PBC}$ with intention, $\mathrm{PBC}$ and intention with behaviour) and large (attitude with intention) in magnitude. When considered together, the associations between TPB variables and food choice behaviours were similar to those reported by McEachan et al. [13]. However, when individual behaviours were coded into distinct categories, we found differences with important theoretical and practical implications.

\section{Moderation by type of food choice behaviour}

As per TST, one might have expected the intention to choose highly palatable, energy-dense foods to have stronger associations with behaviour, as these food choices are typically supported by strong positive immediate contingencies to consume compared to 'healthy'

Table 2 Random-effects average correlation and heterogeneity statistics for TPB associations and healthy eating

\begin{tabular}{lllllll}
\hline Association & $n$ & $k$ & $r_{+}$ & $\mathrm{Cl}$ & $Q$ & $P^{2}$ \\
\hline Attitude-intention & 28572 & 54 & 0.54 & $0.49-0.58$ & $1202.19^{* * *}$ & 95.59 \\
Subjective norm-intention & 28572 & 54 & 0.37 & $0.33-0.42$ & $988.66^{* * *}$ & 94.63 \\
PBC-Intention & 28284 & 53 & 0.42 & $0.36-0.48$ & $2015.34^{* * *}$ & 97.42 \\
PBC-Behaviour & 28737 & 54 & 0.27 & $0.23-0.32$ & $843.16^{* * *}$ & 93.71 \\
Intention-Behaviour & 29465 & 60 & 0.45 & $0.40-0.49$ & $1326.56^{* * *}$ \\
\hline
\end{tabular}

$n=$ number of participants, $k=$ number of effect sizes included in the analysis, $\mathrm{Cl}=95 \%$ confidence interval, $Q$ and $l^{2}=$ tests of heterogeneity, $r_{+}=$random effects average correlation, ${ }^{* * *} p<.001$ 
Table 3 Random-effects average correlation and heterogeneity statistics by category of behaviour

\begin{tabular}{|c|c|c|c|c|c|c|c|c|c|c|c|c|}
\hline \multirow[b]{3}{*}{ Association } & \multicolumn{8}{|c|}{ Health promoting food choice behaviours } & \multirow{2}{*}{\multicolumn{4}{|c|}{$\begin{array}{l}\text { Health compromising food } \\
\text { choice behaviours }\end{array}$}} \\
\hline & \multicolumn{4}{|c|}{ Consume health promoting } & \multicolumn{4}{|c|}{ Avoid health compromising } & & & & \\
\hline & n & k & $r_{+}$ & $P^{2}$ & n & k & $r_{+}$ & $P^{2}$ & n & $k$ & $r_{+}$ & $P^{2}$ \\
\hline PBC-Behaviour & 6413 & 19 & 0.35 & 92.56 & 6232 & 7 & 0.21 & 87.71 & 8726 & 17 & 0.17 & 93.22 \\
\hline Intention-Behaviour & 7676 & 21 & 0.43 & 87.98 & 6518 & 10 & 0.28 & 90.16 & 9013 & 18 & 0.45 & 94.87 \\
\hline
\end{tabular}

$n=$ number of participants, $k=$ number of effect sizes included in the analysis, $\mathrm{Cl}=95 \%$ confidence interval, $l^{2}=$ tests of heterogeneity, $r_{+}=$random effects average correlation

food choices where immediate contingencies are few [15]. However, when we examined differences between these behaviours from the perspective of TST, we failed to find an expected difference for the intentionbehaviour association. We did, however, find that health promoting foods had a significantly higher PBCbehaviour association than health compromising foods. According to the TPB, PBC influences behaviour directly to the extent that it reflects actual control [34]. It may be, therefore, that people have inaccurate perceptions of their control over their decision to consume health compromising foods. Alternatively, given how easy it is for people to make health compromising food choices due to supportive immediate contingencies, it could be that perceptions of control are less relevant. This finding stands in contrast with previous research that has reported negative $\mathrm{PBC}$-behaviour associations for health compromising behaviours [23,35].

When health promoting foods were examined from the perspective of dual-process models of behaviour, we found that there was evidence of moderation both for the PBC-behaviour and intention-behaviour associations. In both cases, mean correlations were significantly larger for those studies examining choosing health promoting foods, as opposed to avoiding health compromising foods. This suggests that the determinants of avoiding health compromising foods are less well captured by reflective, self-report measures than behaviours related to more deliberate, goal-oriented behaviour such as choosing foods in order to achieve health gains. Resisting the impulse to eat highly palatable, health compromising foods is likely to be explained better using assessment strategies designed to tap into non-reflective determinants of behaviour, for example using the Implicit Association Test (IAT, [36]).

\section{Moderation by participant characteristics}

Intentions were more weakly associated with behaviour for those participants who were aged 17 or younger compared to the oldest age group. This mirrors the results reported for dietary behaviours by McEachan et al. [13], who also found $\mathrm{PBC}$ to have stronger associations with behaviour in older compared to younger age groups, a trend not apparent here. Previous studies have suggested that the TPB may be more weakly associated with food choice and other consumption behaviours in younger adults due to their being more likely to live at home and thus less likely to fully determine what they eat or drink (e.g. [23, 37]). We did not find any evidence that associations were moderated by participants' gender. This is in spite of research suggesting that men and women differ in their dietary patterns $[8,19]$. It is possible that our method of testing for the effects of gender, using the proportion of female participants in the sample, may have masked any true differences. It may instead have been preferable to use the method of Cooke et al. [23] who looked at differences between all-male, all-female and combined samples, however there were insufficient studies to conduct the analysis in this way (see Table 1). Further examination of this issue may be warranted.

\section{Strengths and limitations}

Strengths of the current review include the broad search strategy, targeting both published and unpublished research and the use of established criteria [21] to guide

Table 4 Random-effects average correlation and heterogeneity statistics by age of participants

\begin{tabular}{|c|c|c|c|c|c|c|c|c|c|c|c|c|}
\hline \multirow[b]{2}{*}{ Association } & \multicolumn{4}{|l|}{$\leq 17$} & \multicolumn{4}{|l|}{$18-29$} & \multicolumn{4}{|l|}{$\geq 30$} \\
\hline & $n$ & $k$ & $r_{+}$ & $P^{2}$ & $n$ & k & $r_{+}$ & $P^{2}$ & $n$ & $k$ & $r_{+}$ & $P^{2}$ \\
\hline Attitude-intention & 6238 & 8 & 0.46 & 93.13 & 3149 & 9 & 0.41 & 71.52 & 3583 & 11 & 0.55 & 92.82 \\
\hline SN-intention & 6238 & 8 & 0.35 & 95.69 & 3149 & 9 & 0.32 & 85.62 & 3583 & 11 & 0.33 & 82.86 \\
\hline PBC-Intention & 6238 & 8 & 0.41 & 95.63 & 3149 & 9 & 0.55 & 95.50 & 3583 & 11 & 0.45 & 91.62 \\
\hline PBC-Behaviour & 5975 & 6 & 0.21 & 92.39 & 3213 & 10 & 0.34 & 89.07 & 3475 & 10 & 0.35 & 91.70 \\
\hline Intention-Behaviour & 6309 & 10 & 0.26 & 92.68 & 3213 & 10 & 0.42 & 74.86 & 3576 & 11 & 0.44 & 89.08 \\
\hline
\end{tabular}

$n=$ number of participants, $k=$ number of effect sizes included in the analysis, $\mathrm{Cl}=95 \%$ confidence interval, $l^{2}=$ tests of heterogeneity, $r_{+}=$random effects average correlation. NB Restricted to health promoting food choice behaviours only 
the design, conduct and reporting of the meta-analysis. The review is limited, however, by the high level of heterogeneity reported for all associations. This is in spite of the inclusion of a sample of studies that were largely homogenous in terms of participants, behaviour and theoretical background. Finally, there is a further limitation in that the assessment of behaviour within many of the studies examined is crude, with very few studies recording actual dietary intake (see Additional file 5).

\section{Practical implications}

Although the medium-to-large mean associations between TPB variables and behaviour identified in the current review suggest this model may provide a solid foundation for interventions to increase rates of health promoting food choices, any recommendation must be accompanied by some important caveats.

First, the current analyses strongly suggest that the rational variables captured by the $\mathrm{TPB}$ are more strongly associated with some food choices than others. It is imperative that researchers carefully consider the nature of the behaviour under examination prior to selecting a theory to predict that behaviour. The concept of a behavioural diagnosis is central to key frameworks for understanding the determinants of behaviour to inform intervention design [38]. The same considerations should be applied when selecting theory to use in observational research seeking to understand health behaviours.

Second, designers of interventions aiming to reduce the consumption of unhealthy foods should consider alternative approaches not reliant on changing rational determinants of behaviour such as those described in the TPB. Potential approaches include negative evaluative conditioning, training to withhold responses to tempting stimuli and the formation of implementation intentions $[39,40]$. These interventions aim to help individuals counter the automatic impulses to consume that can arise when highly palatable, health compromising foods are encountered. It is worth noting, however, that even in cases where strong associations between the TPB and behaviour are suggested by the current review (e.g. with health promoting food choices), the utility of the model as a basis for developing effective interventions has not been supported by the evidence [41].

Third, it is important to remember that whilst the evidence presented here are informative, ultimately they provide an incomplete record of the potential of the TPB as a medium for behaviour change. This is due primarily to two reasons: (1) that the associations revealed in observational studies are not always reflected in experimental research [42]; and (2) the observational nature of the findings presented here leave open the possibility that the relationship is spurious and that an unmeasured variable has a causal impact on both TPB variables and behaviour. Experimental research aiming to facilitate a change in food choice behaviours by targeting TPB variables is clearly warranted.

\section{Conclusions}

There is a clear imperative for the design of interventions to maximise the health and wellbeing populations by increasing rates of health promoting food choices and concurrently discouraging health-compromising food choices. Understanding the key determinants of these behaviours can assist in the development of such interventions. Based on the evidence presented here, the potential of the TPB as a model either to understand these behaviours or serve as a basis for intervention development, appears limited. Although we found that TPB variables had medium to large associations both with intention and behaviour, the associations between key variables and behaviour were significantly lower both for choosing and avoiding health compromising foods, and in younger age groups. The current review reinforces the complex nature of dietary behaviour and the factors that underpin individual food choices and highlights the need to consider alternative models and determinants of behaviour.

\section{Additional files}

Additional file 1: PRISMA Checklist. (DOCX $26 \mathrm{~kb}$ )

Additional file 2: Electronic search strategies. (DOCX $13 \mathrm{~kb}$ )

Additional file 3: Glossary of key terms and definitions. (DOCX $21 \mathrm{~kb}$ )

Additional file 4: Forest plots for associations between TRA/TPB variables and behaviour. (DOCX $541 \mathrm{~kb}$ )

Additional file 5: Details of method used to measure behaviour in each study. (DOCX $19 \mathrm{~kb}$ )

Competing interests

The authors declare that they have no competing interests.

Authors' contributions

MsMcD designed and executed the search strategies for the review, selected studies, planned and conducted the analyses and drafted the manuscript. MO selected studies, extracted data, coded studies and helped to draft the manuscript. AS extracted data and coded studies. TS \& EJB contributed to the analysis and helped to draft the manuscript. TC, DI \& PC conceived and designed the study and helped to draft the manuscript. RS conceived and designed the study and analysis, provided oversight to co-ordinated the project and helped to draft the manuscript. All authors read and approved the final manuscript.

\section{Acknowledgements}

We thank Megan Andrews for help in screening the literature and data extraction. We also thank Murad Safadi for designing and maintaining the database used to handle extracted data. This research was supported under the Australian Research Council's Discovery Projects funding scheme (project number: DP130100068) awarded to Professors Sharma, Iverson and Coltman. The ARC had no role in the collection, analysis and interpretation of data, or the right to approve the finished manuscript prior to publication. 


\section{Author details}

${ }^{1}$ School of Computing and Information Technology, Faculty of Engineering and Information Sciences, University of Wollongong, Wollongong, NSW 2522, Australia. ${ }^{2}$ School of Medicine, Faculty of Science, Medicine and Health, University of Wollongong, Wollongong, NSW 2522, Australia. ${ }^{3}$ School of Management, Operations and Marketing, Faculty of Business, University of Wollongong, Wollongong, NSW 2522, Australia. ${ }^{4}$ Faculty of Health, Arts and Design, Swinburne University of Technology, Melbourne, VIC 3122, Australia. ${ }^{5}$ School of Psychology, Faculty of Social Sciences, University of Wollongong, Wollongong, NSW 2522, Australia. ${ }^{6}$ Centre for Health and Social Research (CHaSR), Australian Catholic University, Melbourne, Victoria, Australia.

Received: 22 September 2015 Accepted: 19 December 2015 Published online: 30 December 2015

\section{References}

1. World Health Organisation. The top 10 causes of death. World Health Organisation. 2014. http://www.who.int/mediacentre/factsheets/fs310/en/. Accessed 04/05/15.

2. Bloom DE, Cafiero ET, Jané-Llopis E, Abrahams-Gessel S, Bloom LR, Fathima $S$, et al. The global economic burden of noncommunicable diseases. Geneva: World Economic Forum; 2011.

3. World Health Organisation. Global status report on noncommunicable diseases 2010. Geneva: World Health Organisation; 2011.

4. National Health and Medical Research Council. Australian dietary guidelines. Canberra: National Health and Medical Research Council; 2013.

5. National Health Service. Eight tips for healthy eating; 2014. http://www.nhs. uk/Livewell/Goodfood/Pages/eight-tips-healthy-eating.aspx. Accessed 04/ 09/2015 2015

6. U.S. Department of Agriculture and U.S. Department of Health and Human Services. Washington DC: Dietary Guidelines for Americans, 2010; 2010.

7. Jacobs DR, Tapsell LC. Food, not nutrients, is the fundamental unit in nutrition. Nutr Rev. 2007;65(10):439-50.

8. Australian Bureau of Statistics. Australian health survey: nutrition first results. Canberra: Food and Nutrients, 2011-12; 2014.

9. Michie S, Johnston M. Theories and techniques of behaviour change: developing a cumulative science of behaviour change. Health Psychol Rev. 2012;6(1):1-6. doi:10.1080/17437199.2012.654964.

10. Ajzen I. The theory of planned behavior. Organ Behav Hum Dec. 1991;50(2): 179-211. doi:10.1016/0749-5978(91)90020-T.

11. Fishbein M, Ajzen I. Belief, attitude, intention and behaviour: an introduction to theory and research. Reading MA: Addison-Wesley; 1975.

12. Ajzen I. From intentions to actions: A theory of planned behavior. In: Kuhl J, Beckman J, editors. Action-control: From cognition to behavior Heidelberg: Springer; 1985. p. 11-39.

13. McEachan RRC, Conner M, Taylor NJ, Lawton RJ. Prospective prediction of health-related behaviours with the Theory of Planned Behaviour: a metaanalysis. Health Psychol Rev. 2011;5(2):97-144. doi:10.1080/17437199.2010. 521684

14. Povey R, Conner M, Sparks P, James R, Shepherd R. Application of the Theory of Planned Behaviour to two dietary behaviours: roles of perceived control and self-efficacy. Br J Health Psychol. 2000:5:121-39.

15. Hall PA, Fong GT. Temporal self-regulation theory: a model for individual health behaviour. Health Psychol Rev. 2007;1(1):6-52

16. Collins A, Mullan B. An extension of the theory of planned behavior to predict immediate hedonic behaviors and distal benefit behaviors. Food Qual Prefer. 2011;22(7):638-46.

17. Hofmann W, Friese M, Wiers RW. Impulsive versus reflective influences on health behavior: a theoretical framework and empirical review. Health Psychol Rev. 2008;2(2):111-37.

18. Strack F, Deutsch R. Reflective and impulsive determinants of social behavior. Pers Soc Psychol Rev. 2004;8(3):220-47. doi:10.1207/s15327957pspr0803_1.

19. World Health Organisation. Comparative quantification of health risks: Global and regional burden of disease attributable to selected major risk factors. Geneva: World Health Organisation; 2004.

20. Moher D, Liberati A, Tetzlaff J, Altman DG. Preferred reporting items for systematic reviews and meta-analyses: the PRISMA statement. PLoS Med. 2009;6(7), e1000097. doi:10.1371/journal.pmed.1000097.

21. Moher D, Tricco AC. Issues related to the conduct of systematic reviews: a focus on the nutrition field. Am J Clin Nutr. 2008;88(5):1191-9.
22. McDermott MS, Oliver M, Simnadis T, Beck EJ, Coltman T, Iverson D, et al. The Theory of Planned Behaviour and dietary patterns: a systematic review and meta-analysis. Prev Med. 2015;81:150-6. doi:10.1016/j.ypmed.2015.08.020.

23. Cooke R, Dahdah M, Norman P, French DP. How well does the theory of planned behaviour predict alcohol consumption? A systematic review and meta-analysis. Health Psychol Rev. 2014:1-20. doi:10.1080/17437199.2014.947547.

24. Landis JR, Koch GG. The measurement of observer agreement for categorical data. Biometrics. 1977;33(1):159-74.

25. de Bruijn GJ, van den Putte B. Adolescent soft drink consumption, television viewing and habit strength. Investigating clustering effects in the Theory of Planned Behaviour. Appetite. 2009;53(1):66-75. doi:10.1016/j.appet.2009.05.008.

26. Borenstein M, Hedges LV, Higgins JPT, Rothstein HR. Introduction to metaanalysis. Chichester: John Wiley \& Sons; 2009.

27. Sharma R, Yetton P, Crawford J. Estimating the effect of common method variance: the method-method pair technique with an illustration from TAM research. MIS Q. 2009;33(3):473-90.

28. Borenstein $M$, Hedges L, Higgins J, Rothstein H. Comprehensive MetaAnalysis. Version 3. Englewood, NJ: Biostat; 2014.

29. Astrom AN, Okullo I. Temporal stability of the theory of planned behavior: a prospective analysis of sugar consumption among Ugandan adolescents. Community Dent Oral Epidemiol. 2004;32(6):426-34.

30. Berg C, Jonsson I, Conner M. Understanding choice of milk and bread for breakfast among Swedish children aged 11-15 years: an application of the Theory of Planned Behaviour. Appetite. 2000;34(1):5-19.

31. Conner M, Hugh-Jones S, Berg C. Using the two-factor Theory of Planned Behaviour to predict adolescent breakfast choices. Educ Child Psychol. 2011; 28(4):37-50.

32. Kassem NO. Predicting milk and soft drink consumption among female adolescents using the theory of planned behavior [Dr.P.H.]. Ann Arbor: Loma Linda University; 2000.

33. Kassem NO, Lee JW, Modeste NN, Johnston PK. Understanding soft drink consumption among female adolescents using the Theory of Planned Behavior. Health Educ Res. 2003;18(3):278-91.

34. Ajzen I, Madden TJ. Prediction of goal-directed behavior - attitudes, intentions, and perceived behavioral-control. J Exp Soc Psychol. 1986;22(5): 453-74. doi:10.1016/0022-1031(86)90045-4.

35. Topa G, Moriano JA. Theory of planned behavior and smoking: meta-analysis and SEM model. Subst Abuse Rehabil. 2010:1:23-33. doi:10.2147/SAR.S15168.

36. Greenwald AG, McGhee DE, Schwartz JLK. Measuring individual differences in implicit cognition: the implicit association test. J Pers Soc Psychol. 1998; 74(6):1464-80. doi:10.1037/0022-3514.74.6.1464.

37. Kothe EJ, Mullan BA. Interaction effects in the theory of planned behaviour: predicting fruit and vegetable consumption in three prospective cohorts. $\mathrm{Br}$ J Health Psychol. 2014. doi:10.1111/bjhp.12115.

38. Michie S, van Stralen MM, West R. The behaviour change wheel: a new method for characterising and designing behaviour change interventions. Implement Sci. 2011;6. doi:10.1186/1748-5908-6-42.

39. Hollands GJ, Prestwich A, Marteau TM. Using aversive images to enhance healthy food choices and implicit attitudes: an experimental test of evaluative conditioning. Health Psychol. 2011;30(2):195-203. doi:10.1037/a0022261.

40. van Koningsbruggen GM, Veling H, Stroebe W, Aarts H. Comparing two psychological interventions in reducing impulsive processes of eating behaviour: effects on self-selected portion size. Brit J Health Psych. 2014; 19(4):767-82. doi:10.1111/bjhp.12075.

41. Hardeman W, Johnston M, Johnston DW, Bonetti D, Wareham NJ, Kinmonth AL. Application of the theory of planned behaviour in behaviour change interventions: a systematic review. Psychol Health. 2002;17(2):123-58. doi:10. 1080/08870440290013644a.

42. Sheeran P, Harris PR, Epton T. Does heightening risk appraisals change people's intentions and behavior? A meta-analysis of experimental studies. Psychol Bull. 2014;140(2):511-43. doi:10.1037/a0033065.

43. Aghamolaei T, Sadat Tavafian S, Madani A. Fish consumption in a sample of people in Bandar Abbas, Iran: application of the theory of planned behavior. Archives of Iranian Medicine. 2012;15(9):545-8.

44. Astrom AN. Validity of cognitive predictors of adolescent sugar snack consumption. Am J Health Behav. 2004;28(2):112-21.

45. Balian A. Influences on school-age children's milk and soft drink intake [Ph. D.]. Ann Arbor: Case Western Reserve University (Health Sciences); 2008.

46. Blanchard CM, Fisher J, Sparling PB, Shanks TH, Nehl E, Rhodes RE, et al. Understanding adherence to 5 servings of fruits and vegetables per day: a theory of planned behavior perspective. J Nutr Educ Behav. 2009;41(1):3-10. 
47. Blanchard CM, Kupperman J, Sparling PB, Nehl E, Rhodes RE, Courneya KS, et al. Do ethnicity and gender matter when using the theory of planned behavior to understand fruit and vegetable consumption? Appetite. 2009; 52(1):15-20.

48. Branscum P, Sharma M. Comparing the utility of the theory of planned behavior between boys and girls for predicting snack food consumption: implications for practice. Health Promot Pract. 2014;15(1):134-40.

49. Brug J, de Vet E, de Nooijer J, Verplanken B. Predicting fruit consumption: cognitions, intention, and habits. J Nutr Educ Behav. 2006;38(2):73-81.

50. Churchill S, Jessop D, Sparks P. Impulsive and/or planned behaviour: Can impulsivity contribute to the predictive utility of the theory of planned behaviour? Br J Soc Psychol. 2008;47(4):631-46.

51. Churchill S, Jessop DC. Reflective and non-reflective antecedents of healthrelated behaviour: exploring the relative contributions of impulsivity and implicit self-control to the prediction of dietary behaviour. Br J Health Psychol. 2011;16(2):257-72.

52. Corry NH. An extended model of the theory of planned behavior: predictive value for risky and preventive weight-related behaviors [Ph.D.]. Ann Arbor: Purdue University; 2008.

53. de Bruijn G-J. Understanding college students' fruit consumption. Integrating habit strength in the theory of planned behaviour. Appetite. 2010;54(1):16-22.

54. de Bruijn GJ, Keer M, van den Putte B, Neijens P. Need for affect, need for cognition, and the intention-fruit consumption relationship: an actioncontrol perspective. Health Educ J. 2012;71(5):617-28. doi:10.1177/ 0017896911409735

55. de Bruijn G-J, Brug J, Van Lenthe FJ. Neuroticism, conscientiousness and fruit consumption: exploring mediator and moderator effects in the theory of planned behaviour. Psychol Health. 2009;24(9):1051-69.

56. de Bruijn G-J, Kremers SP, De Vet E, De Nooijer J, Van Mechelen W, Brug J. Does habit strength moderate the intention-behaviour relationship in the theory of planned behaviour? The case of fruit consumption. Psychol Health. 2007;22(8):899-916

57. de Bruijn G-J, Kremers SP, de Vries H, van Mechelen W, Brug J. Associations of social-environmental and individual-level factors with adolescent soft drink consumption: Results from the SMILE study. Health Educ Res. 2007; 22(2):227-37.

58. de Bruijn G-J, Kremers SP, Schaalma H, van Mechelen W, Brug J. Determinants of adolescent bicycle use for transportation and snacking behavior. Prev Med. 2005;40(6):658-67.

59. Karimi-Shahanjarini A, Rashidan A, Majdzadeh R, Omidvar N, Tabatabai MG, Shojaeezadeh D. Parental control and junk-food consumption: a mediating and moderating effect analysis. J Appl Soc Psychol. 2012;42(5):1241-65.

60. Kassem NO, Lee JW. Understanding soft drink consumption among male adolescents using the theory of planned behavior. J Behav Med. 2004;27(3): 273-96. doi:10.1023/B:Jobm.0000028499.29501.8f

61. Kassem NO, Lee JW. Understanding reduced-fat milk consumption among male adolescents using the theory of planned behavior. Am J Health Educ. 2005;36(1):16-24.

62. Kida I, Åstrøm A. Correlates of the intention to avoid intake of sugared snacks among Tanzanian adolescents. J Gend Cult Health. 1998;3(3):171-82. doi:10.1023/a:1023241222619.

63. Kim K, Reicks M, Sjoberg S. Applying the Theory of Planned Behavior to predict dairy product consumption by older adults. J Nutr Educ Behav. 2003;35(6):294-301.

64. Mahon D, Cowan C, McCarthy M. The role of attitudes, subjective norm, perceived control and habit in the consumption of ready meals and takeaways in Great Britain. Food Qual Prefer. 2006;17(6):474-81. doi:10.1016/ j.foodqual.2005.06.001.

65. Masalu J, Astrom A. Predicting intended and self-perceived sugar restriction among Tanzanian students using the theory of planned behavior. J Health Psychol. 2001;6(4):435-45.

66. Mitterer-Daltoe ML, Latorres JM, Queiroz MI, Fiszman S, Varela P. Reasons underlying low fish consumption where availability is not an issue. A case study in Brazil, One of the World's largest fish producers. J Sens Stud. 2013; 28(3):205-16. doi:10.1111/joss.12037.

67. Murnaghan DA, Blanchard CM, Rodgers WM, LaRosa JN, MacQuarrie CR, MacLellan DL, et al. Predictors of physical activity, healthy eating and being smoke-free in teens: a theory of planned behaviour approach. Psychol Health. 2010;25(8):925-41.
68. O'Neal CW, Wickrama K, Ralston PA, llich JZ, Harris CM, Coccia C, et al. Eating behaviors of older African Americans: an application of the Theory of Planned Behavior. Gerontologist. 2014;54(2):211-20. doi:10.1093/geront/gns155.

69. Onwezen MC, Bartels J, Antonides G. The self-regulatory function of anticipated pride and guilt in a sustainable and healthy consumption context. Eur J Soc Psychol. 2014;44(1):53-68.

70. Prell H, Berg C, Jonsson L. Why dont adolescents eat fish? Factors influencing fish consumption in school. Scand J Nutr. 2002;46(4):184-91.

71. Richetin J, Perugini M, Adjali I, Hurling R. Comparing leading theoretical models of behavioral predictions and post-behavior evaluations. Psychol Market. 2008;25(12):1131-50. doi:10.1002/Mar.20257.

72. Sharifirad G, Yarmohammadi P, Azadbakht L, Morowatisharifabad MA, Hassanzadeh A. Determinants of fast food consumption among Iranian high school students based on planned behavior theory. J Obes. 2013;2013: 147589.

73. Sjoberg SA. The influence of sensory and behavioral factors on whole grain bread consumption among a convenience sample of adults [Ph.D.]. Ann Arbor: University of Minnesota; 2012.

74. Tak N, Te Velde S, Oenema A, Van der Horst K, Timperio A, Crawford D, et al. The association between home environmental variables and soft drink consumption among adolescents. Exploration of mediation by individual cognitions and habit strength. Appetite. 2011;56(2):503-10.

75. Tak NI, te Velde SJ, Kamphuis CB, Ball K, Crawford D, Brug J, et al. Associations between neighbourhood and household environmental variables and fruit consumption: exploration of mediation by individual cognitions and habit strength in the GLOBE study. Public Health Nutr. 2013; 16(3):505-14.

76. Towler G, Shepherd R. Modification of Fishbein and Ajzen's theory of reasoned action to predict chip consumption. Food Qual Prefer. 1991;3(1): 37-45. http://dx.doi.org/10.1016/0950-3293(91)90021-6.

77. Tuu HH, Olsen SO, Thao DT, Anh NTK. The role of norms in explaining attitudes, intention and consumption of a common food (fish) in Vietnam. Appetite. 2008:51(3):546-51.

78. Verbeke W, Vackier I. Individual determinants of fish consumption: application of the theory of planned behaviour. Appetite. 2005;44(1):67-82.

79. Verplanken B. Beyond frequency: habit as mental construct. Br J Soc Psychol. 2006:45(3):639-56.

80. Zoellner J, Estabrooks PA, Davy BM, Chen Y-C, You W. Exploring the theory of planned behavior to explain sugar-sweetened beverage consumption. J Nutr Educ Behav. 2012:44(2):172-7.

\section{Submit your next manuscript to BioMed Central and we will help you at every step:}

- We accept pre-submission inquiries

- Our selector tool helps you to find the most relevant journal

- We provide round the clock customer support

- Convenient online submission

- Thorough peer review

- Inclusion in PubMed and all major indexing services

- Maximum visibility for your research

Submit your manuscript at www.biomedcentral.com/submit 